\title{
Time estimation and expectancies
}

\author{
MARILYN G. BOLTZ \\ Haverford College, Haverford, Pennsylvania
}

\begin{abstract}
The purpose of this research was to examine the influence of different types of expectancies on time estimation behavior. In Experiment 1, subjects were led to expect that a given number of trials would occur in a music perception task. In Experiment 2, expectancies concerning the duration of a given waiting period were varied. Results of both experiments indicated that the confirmation/violation of expectancies exerted a significant impact on duration judgments. When subjects received more/fewer trials than expected or waited for a duration that was longer/shorter than expected, the total duration of these time spans was over- and underestimated, respectively. Conversely, time estimates were relatively accurate when subjects' expectancies were confirmed. The results of Experiment 1 further revealed that reaction time responses within the music perception task were also systematically influenced by the expected amount of activity. Results are discussed in terms of a framework that emphasizes the role of anticipatory attending on time estimation behavior.
\end{abstract}

In everyday behavior, there are many situations in which we judge the duration of a given event or how much time was spent performing a particular activity. A primary goal of time estimation research is to determine the factors that result in the relative accuracy of these estimates and/or systematic distortions wherein the relevant time span is either under- or overestimated. Some of these factors are assumed to involve certain constitutional states within an individual (e.g., stress and arousal, aging, drugs), whereas others are assumed to arise from the environmental context itself (e.g., the complexity or familarity of an event, task demands). One variable, however, that has received relatively little empirical attention in volves the expected ending time of the event or activity to be judged. That is, the various events and activities that fill our daily lives are typically demarcated from one another by a nonarbitrary beginning and ending point. And we often can anticipate when in time these events and activities will end, so that we can move on to something new. These types of temporal expectancies can stem from multiple sources. One is based on prior experience and knowing the temporal sequence of activities that comprise a given behavioral script. For example, most of us know that the ending of the "restaurant script" involves paying the bill and walking out the door. A second source derives from a consensual agreement about how much time or activity will be spent performing a given behavior before its end. For example, a car mechanic may tell us that we will have to wait $45 \mathrm{~min}$ before he or she is finished with the repairs, and a basketball coach may tell

\footnotetext{
This research was supported by a Faculty Research Grant from Haverford College. The author thanks Ron Joyner and Gabriel Dichter for the collection of data and assistance with the data analyses. Requests for reprints should be addressed to M. Boltz, Department of Psychology, Haverford College, Haverford, PA 19041 (e-mail: mboltz@acc. haverford.edu.)
}

the players that they will have to run 10 more laps around the gym before practice is over. A final source of expected ending times is based on the immediately unfolding context of an event itself. Many events, such as speech or music, display a high degree of temporal regularity such that listeners can extrapolate the preceding context and anticipate when in time an utterance or melody will end. This, in turn, ensures the smooth exchange of speaking roles in interactive speech or the flow from one lead instrument to another during a concert performance (Jaffe \& Feldstein, 1972; Rasch, 1979, 1988).

Despite these different manifestations of temporal expectancies in everyday behavior, very little research has systematically investigated their impact on time estimation judgments. One exception is the study of expectant waiting within the context of the "watched-pot phenomenon." When subjects are asked to signal when a beaker of water begins to boil, this time period is significantly overestimated relative to a comparable interval in which no such expectancy is induced (Block, George, \& Reed, 1980; Cahoon \& Edmonds, 1980). Similar sets of findings have been reported by others who have used a variety of techniques to induce conditions of expectant waiting (e.g., Edmonds, Cahoon, \& Bridges, 1981; Filer \& Meals, 1949; Hare, 1963; Schiff \& Thayer, 1968).

This general phenomenon has been interpreted from at least two different theoretical perspectives. One is offered by Fraisse $(1963,1984)$, who claims that waiting, and expectancies in general, draw attention to the passage of time and thereby increase experienced duration. When attention is directed away from time's passage and toward event or task characteristics, experienced duration decreases and time appears to transpire more quickly. A second intepretation stems from Ornstein's (1969) "storage-size hypothesis." From this perspective, expectancies for an upcoming event increase one's vigilance level and overall alertness. This in turn increases the amount of informa- 
tion stored in memory such that experienced duration is lengthened.

Although these two approaches both offer very reasonable accounts of the watched-pot phenomenon, it is important to note that they have exclusively focused on expectancies concerning the upcoming "what" of an eventnamely, waiting for a pot to boil or some other type of event to occur. While these types of expectancies can and do arise in everyday behavior, it is also the case that one can frequently anticipate when in time a future event will occur. As mentioned earlier, expected ending times can be extrapolated from an underlying behavioral script, a consensual agreement, or the unfolding context of the event itself. In the watched-pot phenomenon, these types of temporal expectancies are missing because the onset of boiling is dependent on the stove's temperature and the amount of water in the pot, and so the upcoming ending point cannot be reliably predicted. However, in order to thoroughly understand the potential impact of expectancies on time estimation behavior, anticipations for both the upcoming "what" and "when" of an event need to be addressed.

One approach that attempts to do this has recently been offered by Jones and Boltz (1989). As described in the following section, this expectancy/contrast model was originally developed to address expected ending times stemming from the immediately unfolding context of a dynamic event. However, given the model's underlying set of assumptions and predictions, it may also have relevance to other ecological contexts in which temporal expectancies derive from alternative sources.

\section{An Expectancy/Contrast Model of Judged Duration}

The theoretical framework proposed by Jones and Boltz (1989) differs from previous models in that it attempts to address a wide range of events that are typically encountered in the natural environment. The basic idea is that natural events vary in the coherence and predictability of their internal structure which, in turn, leads to different selective attending modes that ultimately influence the use of different time estimation strategies. Some of these strategies involve anticipatory attending and the generation of structurally based expectancies; others involve constructs offered by previous time estimation models.

Many events within the natural environment can be described as highly coherent in that they transpire over a given time span marked by a nonarbitrary beginning and end. Within this total time span, the layout of the event's temporal and nontemporal information can be demonstrated to bear lawful relations to one another that, in many instances, approximates a hierarchical arrangement. For example, much of Western music can be characterized as a nontemporal sequence of notes that are nested within melodic phrases and higher order themes and variations, whose ending points are nonarbitrarily marked by tonic triad members specifying the melody's underlying tonal- ity scheme. The inherent rhythmic structure of a tune tends to reinforce this melodic arrangement, in that temporal accents, arising from pauses and prolonged tonal durations, often regularly recur in time to coincide with phrase ending points (e.g., Lerdahl \& Jackendoff, 1983; Yeston, 1976). The result is a joint accent hierarchy in which lower level periodicities (i.e., adjacent notes) are nested within higher order time spans (i.e., melodic phrases) that bear a ratio relationship to one another. In addition, both the temporal and the nontemporal information within this hierarchy are inextricably entwined with one another: the hierarchical nesting of temporal periodicities is defined by different levels of pitch change, and the nesting of pitch changes themselves is defined relative to certain temporal periodicities. This type of structural coherence is not unique to music but can also be found in a variety of other natural events, including speech utterances and conversations (e.g., Grosjean, Grosjean, \& Lane, 1979; Jaffe \& Feldstein, 1972), locomotion patterns (e.g., Carlsoo, 1972; Pierson, 1976; Winter, 1983), various types of environmental sounds (Boltz, 1992), and behavioral action schemes (e.g., Newtson, 1973, 1976).

Since these types of events display a high degree of structural predictability, they are assumed to afford anticipatory attending. Temporal accents marking higher order phrase periodicities serve to capture a perceiver's attention and enable one to effortlessly track an event over time. But more importantly, given that temporal accents coincide with nontemporal ones (e.g., melodic or grammatical phrase boundaries) and both recur with a predictable periodicity, a perceiver is able to extrapolate the event's preceding context and generate expectancies about the what and when of future structural relationships. This involves expectancies about the event's ending because, relative to preceding accents, it too recurs with a predictable periodicity.

According to the model of Jones and Boltz (1989), when one attends in an anticipatory fashion, the confirmation or violation of expected ending times exerts a systematic influence on duration judgments. Let $\mathrm{E}$ stand for the expected ending time of an event that is extrapolated from its immediately unfolding context. This expected ending point serves to define the event's total time span and provides a cognitive referent toward which all ongoing activity is directed. Given this, any discrepancy between the event's actual and expected ending time will thereby be attentionally salient and correspond to a momentary "surprise" in the perceiver. These momentary surprises are formalized as temporal contrasts and represent signed differences between expected and observed ending times $(\mathrm{O}-\mathrm{E})$. In terms of judged duration, the gist of the model is this: if an event's ending confirms expectancies by occurring "on time" (i.e., zero contrast), then duration estimates will be fairly accurate. However, events ending later than expected (i.e., positive temporal contrast) will seem relatively long and thus yield overestimations of judged duration. Conversely, events ending earlier than expected (i.e., negative temporal contrast) will seem rela- 
tively short and thereby yield underestimations. In sum, when individuals rely on an anticipatory mode of attending, duration estimates are assumed to depend on temporal contrast that arises from a temporal disparity between an event's actual and expected ending time.

Although many natural events are structurally coherent, others are less so (see Jones \& Boltz, 1989, for a more detailed analysis). This is most apparent in unskilled productions. Consider, for example, the music of a novice pianist or the speech of a foreigner learning a new language where temporal accents (i.e., pauses or prolonged durations) often fail to coincide with grammatical phrase relations. Since these types of events lack predictability and nested levels of structure, they fail to support anticipatory attending and the generation of expectancies. Instead, attending is directed toward lower order relations and the event's level of detail where people will be more attuned to changes among adjacent items (e.g., changes in successive notes or words) or local groupings of items based on a common structural attribute. When inferring relative duration estimates, people may then simply count and compare the total number of changes or groups within the events themselves. This type of strategy, in fact, has been proposed in previous models of time estimation behavior. For example, Ornstein's (1969) storage-size hypothesis claims that duration estimates are inferred from the number of "chunks" stored in memory, while Block's $(1985,1989)$ cognitive change hypothesis assumes that experienced duration increases with the number of contextual changes. Block's model, in particular, has accrued impressive empirical support (see Block, 1989, for a review) and is able to address a wide range of time estimation phenomena.

In sum, the expectancy/contrast model of Jones and Boltz (1989) is not incompatible with previous theoretical frameworks, but simply extends their overall scope by considering a wider range of natural event structure and the impact of different attending modes upon time estimation behavior. Highly coherent events support anticipatory attending where duration estimates depend on temporal contrast arising from the confirmation/violation of expectancies. Conversely, less coherent events encourage a more analytical mode of attending where duration estimates are based on the relative number of chunks or changes within an event.

Some support for these ideas was obtained in a series of studies relying on musical events as experimental stimuli. Boltz (1989) found that when subjects compare the relative duration of folk tunes varying in tonal resolution, melodies ending on the leading tone interval (i.e., the penultimate note of the underlying scale) are judged shorter than those ending on the conventional tonic. Because the leading tone leaves a listener "hanging" before the expected return to tonicity (Kramer, 1982; Meyer, 1956), such endings seem too early in time and thus yield underestimations. In other experiments, expectancies arising from the regular accentuation of phrase ending points have been examined (Boltz, 1985; Jones \& Boltz, 1989; Jones, Boltz, \& Klein, 1993). Subjects were presented with melodies that supported anticipatory attending in that temporal accents always coincided with melodic phrase boundaries and, at least initially, always recurred after an invariant number of beats. However, in some versions of the melodies, the duration of the final phrase was manipulated such that the last note either appeared to occur "too early" or "too late" relative to the preceding phrase periodicities. Results indicated that the relative duration of these tunes were under- and overestimated, respectively, even though all melodies were equivalent in total duration and total number of beats. Lastly, Boltz (199 lb) has replicated these findings and found that the same event can lead to different time estimation strategies, depending on which level of structure is attended to and the degree of coherence within that level

The primary goal of the present research is to assess the generality of these results and the overall scope of the expectancy/contrast model. It is motivated by several underlying issues that concern the empirical contexts in which the model's assumptions have been evaluated. First, all studies conducted thus far have relied on the prospective paradigm in which subjects know in advance that a time judgment will be required, and may therefore be relying on some sort of conscious time-keeping process (e.g., counting, tapping) as a mediator of this judgment. Although several features of the research argue against such an intepretation (see Jones et al., 1993), it would be useful to adopt a retrospective paradigm in which time judgments are obtained from an incidental memory procedure. This not only reduces the probability that duration estimates will be mediated by factors other than the expectancy construct, but allows one to determine whether effects due to expected ending times persist in immediate memory (i.e., vs. the experience of time in passing). Second, it should be noted that the previous research has all relied on musical events as experimental stimuli. Music is somewhat unique in that most composers rely on a very regularized time structure (i.e., meter, rhythm) to carry a melody, and the use of expectancies for aesthetic expression. Meyer (1956), in particular, has discussed how expected returns to tonal stability are often confirmed to instill a sense of resolution within listeners, but that expectancy violations are just as important for maintaining an audience's interest level. These expectancy violations, in fact, are often temporal in nature such that certain tonal intervals may occur too soon for feelings of surprise or too late for feelings of suspense. Given that expectancy generation is such a critical aspect of musical composition, this raises concerns about whether the previously observed effects are specific to this medium or whether they generalize to other naturalistic contexts. This concern becomes even more legitimate when one considers that expectancies within music are primarily event specific and stem from the immediately unfolding context of a tune. As noted earlier, expected ending times can also 
arise from other sources, and it is therefore of interest to determine whether these too exert an impact upon time estimation behavior.

The two experiments presented here were designed with these issues in mind. Both rely on a retrospective technique and examine the influence of expectancies that derive from a consensual agreement between the experimenter and subjects. In the first experiment, subjects are led to expect that a certain number of trials will occur during the course of the experiment, and these expectancies are either subsequently confirmed or systematically violated. Experiment 2 acted as a converging operation by considering how expectancies for the duration of a given waiting period may affect judged duration.

\section{EXPERIMENT 1}

Much of our everyday behavior can be characterized as goal directed in which a given set of activities is oriented toward a particular ending point (i.e., attaining the goal itself). And we often have an estimate of how much work or activity will be required to achieve this end. For example, in revising a manuscript for publication, we typically have a set of expectancies about which sections of the paper are in need of change, and how much time will be required to make these changes. In many situations, these expectancies are confirmed such that our initial estimates turn out to be quite accurate. In other cases, however, expectancy violations occur in which we discover that either more or less time was required to attain the goal than was previously anticipated.

According to the framework of Jones and Boltz (1989), the relative confirmation and violation of these expectancies may lead to differences in the experienced duration of the activity that was just performed. That is, even though people may spend the same amount of time performing a task to its completion, their experienced duration of this interval may vary according to their initial expectancies. Relative to those individuals who accurately estimate the amount of time required to complete a task, those expending less time than initially anticipated may experience the given time interval as shorter, and those expending more time than anticipated may judge the interval as relatively longer. In the latter two cases, the task's end occurs earlier or later than expected and is therefore predicted to yield underestimations or overestimations, respectively - as has been observed in the actual versus expected ending times of musical compositions.

This hypothesis was tested here in the context of a wellknown phenomenon in the literature, the filled-interval effect. This refers to the finding that two equivalent time intervals are not always judged to have the same duration. Instead, one may seem longer or shorter than the other, depending on the nature of the information filling the intervals. This phenomenon has been well investigated (for reviews, see Allan, 1979; Block, 1979). In fact, it prompted the development of many theories of time estimation behavior. In the present experiment, the basis for the filled-interval effect was established by asking subjects to perform a vigilance task over the course of two blocks of experimental trials that were equivalent in total duration and total number of trials. The task itself was one in which subjects were presented with a set of paired folk tunes and, after hearing a melody in its original composition, were asked to detect an obvious tonal mistake (i.e., a wrong note), which had been introduced during the melody's second presentation. The subjects were told that the speed of these detections was the primary purpose of the experiment, and the upcoming time estimation tasks were not mentioned. After receiving one block of 36 trials (where trials were not numbered in any way), a brief rest break was then provided in which one of three types of expectancies was induced before the presentation of a second block of 36 trials. One group was told that they were halfway through the experiment and would receive one more block of trials before the experiment's end. Given that these subjects subsequently received the same number of trials as experienced earlier, their expectancy about the upcoming amount of activity was thereby confirmed such that the experiment should appear to end on time. In the two remaining groups, however, subjects' expectancies about the upcoming number of trials were subsequently violated. These groups were told that they were either one third or two thirds of the way through the experiment, leading them to expect either twice or one half as many trials as experienced earlier. Given that they actually received the same number of trials as before, they therefore spent less or more time on the task than initially anticipated, such that the experiment's end should seem relatively early or late.

To assess the effects of these manipulations upon time estimation behavior, two types of tasks were administered immediately following the second block of trials. The first was a relative duration judgment in which subjects were asked to decide whether the second block of trials seemed longer, shorter, or the same duration as the first. As a converging operation, the second task required subjects to make an absolute judgment about how much time (in minutes) they thought had transpired between the experiment's beginning and end. If expectancy confirmation/violations exert their predicted effects, then those subjects receiving more (or fewer) trials than expected should judge the second block as relatively longer (or shorter) and overestimate (or underestimate) the experiment's total duration. No such distortion should occur in the expectancy confirmation condition where subjects should not only correctly judge the duration of the second block as identical to that of the first, but should also show more accurate estimates in the absolute judgment task.

There is a final issue of interest that concerns the pattern of reaction time data in the pitch detection task. Some previous research suggests that differences in the overall speed of these responses may vary as a function of the expectancy manipulation. Specifically, Vidacek and Wishner (1971) found that subjects who expect to work for a long period of time show less muscular activity than those 
who expect to work for a shorter period of time. A related study by Walster and Aronson (1967) also found that subjects expecting to continue a series of tasks report less fatigue than do subjects who believe their task is almost over. In effect, people appear to tacitly calibrate their energy level as a function of the expected amount of activity. If these effects generalize to the present study, then it may be the case that subjects receiving more trials than initially anticipated will show slower reaction times in the second block of trials relative to the expectancy confirmation group. Conversely, subjects receiving fewer trials than expected may show very little reduction in the overall speed of responses because their allocated energy level has not yet been depleted.

\section{Method}

Design and Subjects. The experiment was a one-way betweensubjects design. The subjects were led to expect that a given number of trials would occur during the course of the experiment, and these expectancies were subsequently confirmed or violated such that the subjects received the same amount of trials as expected fewer trials than expected or, more trials than expected.

Forty-eight subjects from an introductory psychology course at Haverford College participated in the experiment for course credit. Each had normal hearing abilities and was randomly assigned to one of the three conditions. Although the subjects varied in their degree of musical training and experience, there was an effort to ensure that such differences were evenly distributed across all groups.

Stimulus materials. The experimental stimuli consisted of nine folk tunes (Botsford, 1921), which had been used in a previous study of melody recognition (Boltz, 1993). These had been selected from a music composition book containing folk tunes from other countries and (pre)rated as unfamilar by a group of 12 experienced musicians. An exemplar tune is depicted in Figure 1, and the total set of song titles is presented in the Appendix.

Although all melodies varied in key (C, F, G, or D major), meter (2/4 or $3 / 4$ ), and total number of notes (range of 32 to 39 notes), they were structurally similar in that each contained three melodic phrases of equal duration whose ending points were marked by a tonic triad member (i.e., tonic, mediant, or dominant) that was prolonged in duration (i.e., by a half note, $d$ ). The latter characteristics have been found to increase the memorability of a tune (Boltz, 1991a) and should thereby facilitate the detection of tonal mistakes in the present experiment. For all melodies, tonal duration (on time) and the intertone interval (off time) were always held constant such that all notes corresponded to the following values: half notes $(d)$, $400 \mathrm{msec}$ on, $200 \mathrm{msec}$ off; quarter notes (d), $200 \mathrm{msec}$ on, $100 \mathrm{msec}$ off; and eighth notes $(\curvearrowright), 100 \mathrm{msec}$ on, $50 \mathrm{msec}$ off.

For each of the nine melodies, 8 different notes were replaced with salient pitch deviations that corresponded to an atonal note outside of the melody's key, and a 3- to 6-semitone change that broke the melody's original contour. To a listener, these pitch deviations would sound as if a performer had made a tonal mistake. There was also an attempt to control for the location of pitch devi- ations across the three melodic phrases of a tune in that four occurred at points of high metrical stress and four at points of low stress (see Palmer \& Krumhansl, 1990).

Lastly, melodies were randomized into two blocks, each containing 36 trials. Within each block, an experimental trial consisted of a pair of melodies: a given folk tune in its original composition (i.e., a standard) followed by this same tune containing a single pitch deviation (i.e., a comparison melody). Each of the nine folk tunes was presented four times within a block, where the location of the deviation point varied across successive presentations, both within and between blocks. Half of the pitch deviations within a block always coincided with points of high metrical stress, and the remainder coincided with points of low stress.

Apparatus. All melodies were constructed and generated with the Midilab software system (Todd, Boltz, \& Jones, 1989). During each experimental session, melodies were presented on-line by a Yamaha TX81Z FM tone generator controlled by an IBM-AT computer with a Roland MPU-401 MIDI interface unit. Sequences of tones were amplified by a Kenwood KR-4010 receiver and played over Koss-Pro 4AAA Plus headphones at a comfortable listening level. The timbre of all melodies was that of a percussive flute.

Procedure. The subjects were told that the purpose of the experiment was to determine how quickly they were able to detect tonal mistakes in a set of folk tunes they would be hearing. They were asked to remove all rings, watches, and bracelets so these would not interfere with the speed of their responses. The set of time estimation tasks that were to subsequently follow were not mentioned.

Recorded instructions informed the subjects of pattern presentation details and task requirements. On each trial, a 1 -sec warning tone (C8) preceded a standard melody by $2 \mathrm{sec}$. Two seconds later, the comparison melody of the pair was presented and immediately followed by a 5 -sec period of silence. As they listened to the comparison melody, the subjects were asked to indicate, as quickly as possible, the presence of a tonal mistake (i.e., a wrong note) that would occur at some point within the tune. They were told to make this response by pressing a hand-held button. The response was then automatically recorded by the computer. Trials were not numbered in any way, and the onset of successive trials was simply signaled by a recurrence of the warning tone.

After completing the first block of 36 trials, the subjects were given a 3-min rest break. At the end of this interval, the subjects were told by the experimenter (who was naive to the purpose of the experiment), "We're now (one half) (one third) (two thirds) of the way through the experiment. I'm going to play you one more block of trials and then we'll be finished." The second block of 36 trials was then presented, and the subjects again performed the pitch detection task. Immediately following this, the subjects were given a response sheet and unexpectedly asked to perform two types of time estimation judgments. In the first, they were asked to answer the question (via a circled response), "Did the second block of experimental trials seem longer than, shorter than, or the same duration as the first block of trials?" For the second task, the subjects were asked, "You entered this lab room at__ (actual time was given, e.g., 7:00). What time do you think it is now?" This estimate was written on the provided response sheet.

In each expectancy condition, the subjects were tested in small groups of 2-4 individuals. The total duration of each experimental session was approximately $47 \mathrm{~min}$.

Reapers on the Mountain by E. Leamy

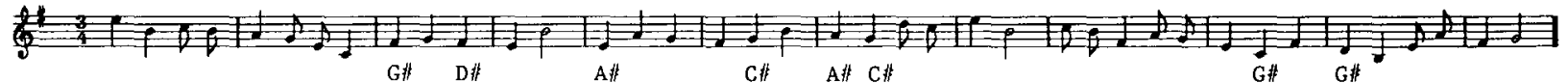

Figure 1. An exemplar melody used in the pitch detection task of Experiment 1. Deviant note values are indicated below the notated staff line; those occurring on the first note of a measure are points of high metrical stress, and those occurring within a measure are points of low metrical stress. 


\section{Results}

Three different measures of time estimation behavior were obtained for all subjects: the frequency of categorical time estimates in the relative duration task, the mean amount of deviation between the actual and judged duration of the experimental session, and mean reaction times in the music perception task. Each is discussed in turn.

Relative duration judgments. For each of the three expectancy conditions, Table 1 depicts the percent frequency in which the duration of the second block of experimental trials was judged longer than, shorter than, or the same as the duration of the first block of trials.

A chi-square analysis revealed a significant effect of the expectancy manipulation $\left(\chi^{2}=38.07, p<.001\right)$. When expectancies for the upcoming number of trials were confirmed, the majority of the subjects $(81 \%)$ correctly judged the second experimental block as identical in duration to the first. This pattern of results, however, markedly altered when expectancies were systematically violated. In Table 1, notice that when the subjects received more trials than expected, $69 \%$ judged the second block as relatively longer than the first. Conversely, most subjects $(75 \%)$ estimated the second block as relatively shorter when they received fewer trials than expected.

In sum, these results confirm predictions of the expectancy/contrast model by indicating that expectancy confirmation leads to accurate time judgments. On the other hand, when subjects do less or more work than expected such that a task appears to end earlier or later than anticipated, then under- and overestimations arise, respectively. The next analysis examines whether similar results occur when subjects are asked to estimate the total duration of the experimental session.

Absolute time estimates. For each subject, the actual duration of an experimental session was subtracted from its judged duration. Those values in the first row of Table 2 therefore reflect the mean amount of error such that positive values indicate overestimations and negative values indicate underestimations.

The overall analysis of variance (ANOVA) revealed that these errors systematically varied as a function of the expectancy manipulation $\left[F(2,45)=17.24, M S_{\mathrm{e}}=2.43\right.$, $p<.001]$. In Table 2, the results show that the subjects in the expectancy confirmation condition slightly (but not significantly) underestimated the total duration of the experimental session by $3 \mathrm{~min}$. The magnitude of this under-

Table 1

Percent Frequency With Which Subjects Judged the Duration of the Second Block of Experimental Trials as Shorter Than,

Longer Than, or the Same as the Duration of the First Block for Each of the Three Expectancy Conditions

\begin{tabular}{cccc}
\hline \multirow{2}{*}{$\begin{array}{c}\text { Number of Actual Trials } \\
\text { Relative to Number of } \\
\text { Expected Trials }\end{array}$} & \multicolumn{3}{c}{ Subjects' Responses } \\
\cline { 2 - 4 } & Shorter & Longer & Same \\
\hline More ("2/3") & 12.5 & 69 & 19 \\
Less ("1/3") & 75 & 6 & 19 \\
Same (" $1 / 2$ ") & 6 & 12.5 & 81 \\
\hline
\end{tabular}

Table 2

Mean Performance in Experiment 1 as a Function of the Amount of Expected Activity

\begin{tabular}{cccccc}
\hline \multirow{2}{*}{$\begin{array}{c}\text { Number of Actual Trials } \\
\text { Relative to Number of }\end{array}$} & \multicolumn{2}{c}{$\begin{array}{c}\text { Judged - Actual } \\
\text { Duration }\end{array}$} & & \multicolumn{2}{c}{$\begin{array}{c}\text { Block 2 RT - } \\
\text { Block 1 RT }\end{array}$} \\
\cline { 2 - 3 } \cline { 5 - 6 } Expected Trials & \multicolumn{1}{c}{$M$} & $S D$ & & $M$ & $S D$ \\
\hline More ("2/3") & +7.37 & 5.91 & +327 & 702 \\
Less ("1/3") & -13.08 & 6.43 & +51 & 686 \\
Same ("1/2") & -3.03 & 2.54 & +124 & 633 \\
\hline
\end{tabular}

Note-For Judged - Actual Duration, $M=$ the mean deviation (in minutes) between subjects' judged duration and the actual duration of the experimental session. Values closer to zero reflect greater accuracy, positive values indicate overestimates, and negative values indicate underestimates. For Block 2 RT - Block 1 RT, $M=$ the mean difference (in milliseconds) in subjects' reaction times to deviant notes between the first and second block of experimental trials. Values closer to zero reflect minimal change in the speed of responses; the positive values indicate that reaction times in Block 2 were relatively longer (i.e., slower). $S D=$ the standard deviation of responses.

estimate, however, significantly increased when the subjects received fewer trials than expected $(M=-13 \mathrm{~min})$. Conversely, significant overestimates occurred when the subjects received more trials than expected (for all Tukey tests, $p<.01$ )

Reaction times. This final analysis examined the subjects' reaction times to the presence of deviant pitch intervals in the music perception task. For each subject, the overall mean response time for the first block of experimental trials was subtracted from that of the second block of trials. The mean deviation score for each expectancy condition is shown in the second row of Table 2, where values closer to zero reflect minimal change in the speed of responses and positive values indicate that reaction times became relatively longer and slower during the second block of trials.

An overall ANOVA showed that performance varied across the three expectancy conditions $[F(2,45)=12.72$, $\left.M S_{\mathrm{e}}=17654.38, p<.001\right]$. In the expectancy confirmation group, the subjects' responses were, on average, 124 msec slower during the second block of trials than in the first $(p<.05)$. In contrast, the subjects receiving fewer trials than expected showed a significantly smaller increase in response latency-a value that, in fact, did not vary from zero (i.e., no change). This finding differs from that for the subjects receiving more trials than expected, where response times were significantly slower during the second block of trials $(p<.01)$.

\section{Discussion}

As a set, this overall pattern of results indicates that the same objective time span can yield relatively accurate estimates, overestimates, or underestimates, depending on the temporal contrast between an event's actual and expected ending time. This effect has previously been observed in the context of musical listening, and it was replicated here with the amount of activity that was expected to occur during an experimental session. This latter finding appears to be a fairly robust one that was observed with several different indices of time estimation behavior. 
The first involved a measure of relative duration in which the subjects were asked to compare the duration of a second block of experimental trials relative to that of a preceding block. Given that these two blocks contained exactly the same number of trials, total duration, and set of stimuli (i.e., melodies), this afforded the opportunity to assess whether expectancies exert a differential impact upon the filled-interval effect. Results indicated that this was the case, such that the subjects expecting more trials than, fewer trials than, or the same number of trials as actually received judged the second block as categorically longer than, shorter than, or the same duration as the first.

A similar pattern of findings was also observed with a verbal estimation task. This second task was quite different from the first in that the subjects were required to produce a value in clock time that represented the total duration of the entire experimental session. Again, results revealed that both accurate and over/underestimates occurred as a function of expectancy confirmation/violation. In addition, there was also some evidence suggesting that the magnitude of this error depended on the magnitude of temporal contrast. That is, the subjects receiving fewer trials than expected displayed greater error than did those receiving more trials than expected (i.e., 13.08 vs. 7.37). This makes sense when one considers that the subjects in the former group were expecting to receive twice as many trials (i.e., 72) in the second versus first block, whereas those in the second group were expecting to receive only one half as many (i.e., 18). The temporal contrast values are therefore -36 versus +18 , and the relative magnitude of these differences is reflected in the data themselves. This effect has also been observed in the context of musical listening (Jones et al., 1993) and suggests that expectancies can also exert a nonordinal influence on behavior.

Lastly, the reaction time data from the pitch detection task also revealed systematic changes as a function of the expectancy manipulation. Overall, the speed of Block 2 versus Block 1 responses became increasingly slower when the subjects thought that the experimental session was either nearing or exceeding its expected ending point. In contrast, the subjects expecting additional trials at the experiment's end showed very little fatigue, such that the overall speed of their responses was comparable to that of the previous block of trials. This finding is consistent with the work of Vidacek and Wishner (1971) and Walster and Aronson (1967), who observed that subjects' muscular activity and subjective reports of fatigue varied with the amount of expected work or physical effort. This calibration of energy resources is typically adaptive in everyday behavior because it ensures that energy will be conserved and not unnecessarily wasted during the course of an activity. However, as the present set of results suggest, the allocation of these resources can sometimes be miscalculated such that activity levels that exceed our initial expectancies can lead to greater fatigue and a slowing down of motor responses.

\section{EXPERIMENT 2}

The second experiment was designed as a converging operation to determine whether the confirmation or violation of expected waiting times also exert systematic effects upon duration estimates. That is, suppose one is waiting for a given time interval before the arrival of a plane or being admitted to see a dentist or doctor. The question addressed here is whether the duration of this time span is differentially experienced according to one's initial expectancies about its relative length. That is, does the duration of, for example, a 15-min time span appear the same regardless if one was initially told that he/she would be waiting for less time than, more time than, or the same amount of time as actually elapsed?

This situation differs from the watched-pot phenomenom in that one has a specific, preconceived idea about how long the waiting period will be. If expectancy confirmations/violations do exert a differential impact, then this, of course, assumes that people have a rough approximation for how long an interval should be. Given our society's dependence on standarized timekeepers, this is not an unreasonable assumption. Our lives' activities, to a large extent, are governed by a clock framework in which appointments, the arrival of planes and trains, and the start of TV programs all adhere to a fairly rigid schedule. Furthermore, when we do wait for the onset of a given event, we often have access to a watch or clock that affords the monitoring of time's passage and the incidental learning of different durational periods, which has been shown to occur for other ecological events (Boltz, 1992). People may therefore have a tacit knowledge about the relative length of different time spans; if one waits for more or less time than expected, then differential time experiences may occur.

This notion was tested here by instructing the subjects that they would have to wait for a particular time period in order for the experiment's computer and apparatus to sufficiently "warm up" for an upcoming music perception task. All subjects actually waited for the same duration but had previously been told that they would be waiting for a time period (in minutes) that was either longer than, shorter than, or identical to this actual duration. When the subjects were later unexpectedly asked to produce an absolute time judgment of the waiting period, it was predicted that overestimates, underestimates, and relatively accurate estimates would occur, respectively.

\section{Method}

Design and Subjects. The experiment was a one-way betweensubjects design in which the subjects waited for a time period that was longer than, shorter than, or the same as initially anticipated. A control group was also included in which the subjects had no specific expectancy for how long the waiting period would be.

Forty-eight subjects from an introductory psychology class at Haverford College participated in the experiment for course credit. Twelve were randomly assigned to one of the four conditions.

Procedure. The subjects were told that they would be participating in a psychomusicology experiment in which they would be hearing 
a set of folk tunes and asked to make a series of perceptual judgments. Because some of these judgments would require a buttonpress on the response console in front of them, they were asked to remove all rings, watches, and bracelets so these would not interfere with the speed of their motor responses. The experimenter, who was naive to the experiment's purpose, then delivered the following line that was intended to induce different expectancies about the upcoming waiting period: "Before the experiment can get underway, we'll need to wait for (a minute; 10 minutes; 20 minutes) in order for the computer and other equipment to sufficiently warm up such that they will later accurately record your data." The subjects in the no-expectancy group were not given a specific time value but were simply told that "we'll need to wait in order for the computer ...." The subjects in all groups then waited for the same 10 -min time period that was unfilled by conversation or the reading of books or other printed material. At the end of this, they were given a sheet of paper and asked to estimate (in minutes) how long they thought they had been waiting for the equipment to warm up. The subjects were then dismissed and told the true purpose of the experiment.

\section{Results and Discussion}

For each subject, the actual duration of the 10 -min waiting period was subtracted from its judged duration. These deviation scores are shown in Table 3 , where values closer to zero reflect greater accuracy, positive values indiate overestimates, and negative values indicate underestimates.

As in Experiment 1, the overall ANOVA revealed that duration judgments vary as a function of expectancies $\left[F(3,44)=18.09, M S_{\mathrm{e}}=2.89, p<.001\right]$. As seen in Table 3, time estimates were most accurate when the expected duration of the waiting period was identical to the actual one-the overall mean error in this condition $(M=$ 1.23 ), in fact, did not significantly vary from zero (i.e., no error). In addition, the results indicate that waiting also led to significant underestimates when the subjects waited for less time than initially anticipated. Only when the subjects waited for a longer interval than anticipated or when they had no idea how long the wait would be did overestimates arise from a lengthening of experienced duration (for all Tukey post hoc comparisons, $p<.01$ ).

\section{GENERAL DISCUSSION}

The present research illustrates that expected ending times exert a marked impact upon time estimation behavior. Although different individuals may engage in the same task or activity for the same amount of time, the duration of this total time span can be differentially ex-

Table 3

Mean Deviation (in Minutes) Between Subjects' Judged Duration and the Actual Duration of the Waiting Period in Experiment 2 as a Function of the Expectancy Conditions

\begin{tabular}{|c|c|c|c|c|c|c|c|}
\hline \multicolumn{2}{|c|}{$\begin{array}{c}\text { Shorter } \\
\text { ("20 min") }\end{array}$} & \multicolumn{2}{|c|}{$\begin{array}{c}\text { Same } \\
\text { ("10 min") }\end{array}$} & \multicolumn{2}{|c|}{$\begin{array}{c}\text { Longer } \\
\text { ("1 min") }\end{array}$} & \multicolumn{2}{|c|}{$\begin{array}{c}\text { No } \\
\text { Expectancy } \\
\end{array}$} \\
\hline$M$ & $S D$ & $M$ & $S D$ & $M$ & $S D$ & $M$ & $S D$ \\
\hline-3.08 & 4.89 & +1.23 & 3.41 & +4.80 & 5.23 & +4.75 & 6.03 \\
\hline
\end{tabular}

Note- $\boldsymbol{M}=$ mean deviation; values closer to zero reflect greater accuracy, positive values indicate overestimates, and negative values indicate underestimates. $S D=$ standard deviation. perienced according to the temporal contrast between its actual and expected ending time. Zero contrast and expectancy confirmations lead to fairly accurate time judgments. However, events ending later than expected yield relative overestimates, and those ending earlier than expected yield relative underestimates.

These findings have previously been observed in the context of musical listening, where subjects were asked to compare the duration of paired melodies that were always equivalent in total duration and number of notes, but varied in their expected ending times. In some cases, these expectancies were based on properties of the final note (Boltz, 1989); in other cases, they arose from the regular accentuation of phrase ending points (Boltz, 1985, 1991b; Jones \& Boltz, 1989; Jones et al., 1993). Both manipulations revealed support for the expectancy/contrast hypothesis and further showed that the magnitude of contrast is associated with a greater magnitude of error.

The two experiments reported here not only converge with these findings but extend them in several different ways. First, they show that effects of temporal contrast are not specific to music but apply to other ecological events. Although composers rely on the confirmation and violation of temporal expectancies for aesthetic expression and the communication of emotional meaning (Meyer, 1956), the attentional focus on expected ending times is also inherent to other naturalistic contexts. Our daily lives, in fact, are filled with a nested sequence of activities and events that are typically demarcated from one another by a nonarbitrary ending point. In many situations, this ending point corresponds to the attainment of a particular goal that may be prescribed by a well-learned behavioral script. Alternatively, an ending point may arise from a consensual agreement among two or more individuals, or even with oneself, about how much activity is to be performed before a task's completion (as in Experiment 1), or the duration of a given waiting period before the onset of an event (as in Experiment 2). Lastly, more transient events, such as speech utterances or melodies, also display nonarbitrary ending points that are marked by certain acoustical changes in the event's structure (e.g., N. Todd, 1985). Across these different instances, ending points serve to define an event's total time span and provide a cognitive anchor toward which all ongoing activity is directed. At the level of the individual, these upcoming ending points can often be anticipated; in fact, it is adaptive to do so. By using the event's expected ending as an internal referent, this not only allows one to gauge and monitor the remaining amount of activity until the event's completion, but it enables one to prepare and plan ahead for the next upcoming event. This ability is particularly useful for the coordination of interactive behavior, such as the exchange of speaking roles or lead instruments in a concert performance, or, more generally, to ensure the smooth transition from one activity to another. In addition, as the present set of results reveals, these internal referents can also play a role in time estimation behavior. When event endings conform to an individual's expectancies, as is 
often the case, then the duration of this time span can be judged quite accurately. Futhermore, as the results of Experiment 1 suggest, one can allocate a sufficient amount of energy such that these resources will not be unnecessarily wasted. When temporal expectancies are violated, errors in judged duration emerge, as well as a misallocation of energy resources that is reflected in the speed of motor responses.

In sum, despite the fact that different types of natural events transpire over different durations and are filled with different types of information, most display a nonarbitrary ending point that serves to demarcate its offset from the onset of another. Assuming that people use the event's expected ending as a referent for gauging their current and planned activities, it is perhaps unsurprising that manipulations of ending points exert similar effects upon behavior, regardless of how these initial expectancies were generated. Thus, even though the expectancy/contrast model of Jones and Boltz (1989) was originally developed to address event-specific expectancies arising from the unfolding context of a dynamic event, its underlying assumptions have relevance to other naturalistic situations.

A second extension of the expectancy/contrast approach involves the nature of the time judgments themselves. Previous tests of the model exclusively relied on a prospective technique wherein subjects knew in advance that a time judgment would be required. This, in turn, allowed for the possibility that time judgments were mediated by some sort of conscious timekeeping strategy (such as counting or tapping), and not the expectancy construct as claimed. In the present experiments, this potential confound was reduced by obtaining incidental measures of time estimation behavior, such that the subjects did not know that duration judgments would be required until after an event had transpired. Although this procedure technically fits the definition of a retrospective paradigm, it should be recognized that this was not entirely so. The subjects in both experiments were given implicit information about the temporal interval they were later asked to judge: in Experiment 1, this information concerned the expected number of trials before the experiment's end, and in Experiment 2, it involved a waiting period of an expected duration (in minutes). On the one hand, it is possible that this information subsequently influenced duration judgments. This is particularly true for Experiment 2, where expectancies, in the form of clock units, might have "anchored" the time judgments and determined the relative magnitude of error. However, it is not clear how the subjects' preknowledge influenced the overall pattern of observed results. For example, if the subjects in Experiment 1 were relying on some type of conscious counting strategy, then the duration of the second experimental block should have been judged identical to the first, because both contained the same number of trials across all expectancy conditions. This, however, was not the case. In addition, it is not clear how induced expectancies influenced the absolute judgment task in both experiments, where it was found that estimates were over/ underestimated relative to the session's actual duration. Lastly, given that the expectancy manipulations were only casually introduced and attention was directed toward the upcoming music perception tasks, there was no reason for the subjects to necessarily anticipate a subsequent time estimation judgment. In sum, there is a need to examine expectancy manipulations in a more "pure" form of the retrospective paradigm. However, the present set of results do suggest that effects of expectancy confirmation/ violation generalize beyond the experience of time in passing and exert an impact on immediate memory.

The underlying assumptions inherent to the expectancy/contrast model are ones that in many ways differ from those of other models; however, on another level, they are not necessarily incompatible with these approaches. The primary difference lies in the range of natural events that is addressed and the emphasis on the way people use natural-event structure for selective attending activities. Previous approaches have focused on the types of events that do not afford anticipatory attending and the generation of expectancies. These are, in fact, quite common in everyday behavior, because activities and circumstances are not always well organized and, therefore, lack an overall level of predictability. In these situations, Jones and Boltz (1989) suggest that people are more apt to rely on the strategies described by Ornstein (1969), Block (1985, 1989), and others, who assert that duration judgments are based on the relative amount of information within an interval. That is, given that one is unable to anticipate what will happen next, an individual's attention is directed toward the unfolding flux of the event where changes among adjacent items or local groupings of items will be highly salient. When inferring relative duration judgments, subjects may then simply count and compare the number of such changes across events. Some support for this idea was observed by Boltz (1991b), who asked subjects to compare the relative duration of paired melodies in which the timing of higher order phrase endings was temporally predictable but lower order contour changes among adjacent notes (i.e., the "ups and downs" of pitch) were not. When forced to attentionally track a given level of structure via buttonpresses, results showed that subjects tracking phrase ending points based their time estimates, not on the relative number of such phrases, but on the confirmation/violation of expected ending times. Conversely, subjects tracking lower level contour changes relied on a counting strategy, wherein melodies containing more changes were judged longer.

This study, then, illustrates that counting strategies are invoked when events lack an overall level of predictability. However, they also show that duration estimates of highly predictable events depend on expected ending times, even when the opportunity to use counting strategies is provided. Many events in the natural environment do, in fact, display a high degree of structural coherence and predictability. For example, in an earlier section of this paper, it was discussed how music, speech, and behavioral action schemes all contain an inherent hierarchi- 
cal organization of information that allows one to extrapolate the event's preceding course and anticipate future structural relations. Similarly, Bower, Black, and Turner (1979) have shown that goal-directed scripts of behavior are perceived to contain a temporal sequence of subaction units whose boundaries are consistently agreed upon. These types of highly structured events not only allow an individual to anticipate the upcoming "what" of the event (i.e., what will happen next), but the upcoming ending time as well. Previous research, such as the watched-pot phenomenon, has examined effects of the first type of expectancy on time estimation behavior. In contexts where people know that something is about to happen but do not know when in time it will occur, it is found that the relevant time span is consistently overestimated. This finding was replicated in the no-expectancy condition of Experiment 2 , in which judged duration was significantly overestimated relative to the wait's actual duration. Fraisse $(1963,1984)$ has attributed this result to an increased awareness to the passage of time per se, whereas Ornstein (1969) has claimed it is due to more information being stored in memory. A third interpretation, not inconsistent with these two viewpoints, is that an increased awareness of time's passage translates into a focal awareness of an event's ending time (i.e., the end of the waiting period itself) and the fact that it has not happened yet. Since the ending in this sense is late in coming, the overall interval may therefore seem long and is overestimated in duration.

More typical, however, are situations in which highly structured events do allow one to predict their upcoming ending point. As the present set of results indicates, the time estimates of these types of events appear to be determined by temporal contrast and the relative confirmation or violation of expectancies-events ending earlier or later than expected yield under- and overestimates, respectively, while those ending on time produce fairly accurate judgments. Overall, then, the assumptions of the expectancy/contrast model are not incompatible with those of alternative approaches. Instead, it appears that people use different types of time estimation strategies, depending on the inherent structure of the events to be judged. Some events support anticipatory attending and the generation of expectancies, while others do not. By examining the effects of both types of events on time estimation behavior, a more integrated theoretical framework thereby results.

In closing, the approach that has been outlined here provides a framework in which to link variations in naturalevent structure to different types of time estimation behavior. It also attempts to incorporate strategies offered by alternative models into a perspective that recognizes a wider range of environmental contexts than has been previously acknowledged. This approach, however, is not intended to pose as an all-encompassing theory of duration judgment. There are a variety of other factors, including those within an individual, that also contribute to temporal experience and yet have not been considered.
Nevertheless, future research and theory development may benefit from an analysis of event structure and its relationship to an individual in order to determine the referents used in time estimation behavior.

\section{REFERENCES}

Allan, L. G. (1979). The perception of time. Perception \& Psychophysics, 26, 340-354

Block, R. A.(1979). Time and consciousness. In G. Underwood \& R. Stevens (Eds.), Aspects of consciousness: Vol. 1. Psychological issues (pp. 179-217). London: Academic Press.

BLock, R. A. (1985). Contextual coding in memory: Studies of remembered duration. In J. Michon \& J. Jackson (Eds.), Time, action, and behavior (pp. 169-178). Heidelberg: Springer.

BLock, R. A. (1989). Experiencing and remembering time: Affordances, context, and cognition. In I. Levin \& D. Zakay (Eds.), Time and human cognition: A life-span perspective (pp. 333-363). Amsterdam: North Holland.

Block, R. A., George, E. J., \& ReED, M. A. (1980). A watched pot sometimes boils: A study of duration experience. Acta Psychologica, 46, 81-94.

Boltz, M. G. (1985). An expectancy model of judged duration: An ecological perspective. Unpublished doctoral dissertation, Ohio State University.

Boltz, M. G. (1989). Time judgments of musical endings: Effects of expectancies in the "filled interval effect." Perception \& Psychophysics, 46, 409-418.

BoLTz, M. G. (1991a). Some structural determinants of melody recall. Memory \& Cognition, 19, 239-251.

BoLTz, M. G. (1991b). Time estimation and attentional perspective. Perception \& Psychophysics, 49, 422-433.

BoLtz, M. G. (1992). The remembering of auditory event durations. Journal of Experimental Psychology: Learning, Memory, \& Cognition, 18, 938-956.

BoLtz, M. G. (1993). The generation of temporal and melodic expectancies during musical listening. Perception \& Psychophysics, 53, $585-600$.

BotsFord, F. H. (Ed.) (1921). Folk songs of many people (Vol. 1). New York: Woman's Press.

Bower, G., Black, J., \& Turner, T. (1979). Scripts in memory for text. Cognitive Psychology, 11, 177-220.

CAHOON, D., Edmonds, E. M. (1980). The watched pot still won't boil: Expectancy as a variable in estimating the passage of time. Bulletin of the Psychonomic Society, 16, 115-116.

CARLsoo, S. (1972). How man moves (W. P. Michael, Trans.). New York: Cran, Russak.

Edmonds, E. M., CAHOon, D., \& Bridges, B. (1981). The estimation of time as a function of positive, neutral, or negative expectancies. Bulletin of the Psychonomic Society, 17, 259-260.

Filer, R. J., \& MEALS, D. W. (1949). The effect of motivating conditions on the estimation of time. Journal of Experimental Psychology, 39, 327-331

Fraisse, P. (1963). The psychology of time. New York: Harper \& Row. FraISSE, P. (1984). Perception and estimation of time. Annual Review of Psychology, 35, 1-36.

Grosjean, R., Grosjean, L., \& LaNe, H. (1979). The patterns of silence: Performance structures in sentence production. Cognitive Psychology, 11, 58-81.

HARE, R. D. (1963). The estimation of short intervals terminated by shock. Journal of Clinical Psychology, 19, 378-380.

JAFFE, J., \& FeldSTEIN, S. (1972). Rhythms of dialogue. New York: Academic Press.

JoNes, M. R., \& BolTz, M. G. (1989). Dynamic attending and responses to time. Psychological Review, 96, 459-491.

Jones, M. R., Boltz, M. G., \& Klein, J. M. (1993). Expected endings and judged duration. Memory \& Cognition, 21, 646-665.

Kramer, J. D. (1982). Beginnings and endings in Western art music. Canadian University Music Review, 3, 1-14. 
LERDAHL, F., \& JACKENDOFF, R. (1983). A generative theory of tonal music. Cambridge, MA: MIT Press.

MEYER, L. B. (1956). Emotion and meaning in music, Chicago: University of Chicago Press.

Newtson, D. (1973). Attribution and the unit of perception of ongoing behavior. Joumal of Personality \& Social Psychology, 28, 28-38

NEWTSON, D. (1976). Foundations of attribution: The perception of ongoing behavior. In J. Harvey, R. Ickes, \& R. Kidd (Eds.), New directions in attribution research (Vol. 1, pp. 223-248). Hillsdale, NJ: Erlbaum.

OrNSteIn, R. E. (1969). On the experience of time. Baitimore, MD Penguin.

Palmer, C., \& Krumhansl, C. L. (1990). Mental representations for musical meter. Journal of Experimental Psychology: Human Perception \& Performance, 16, 728-741.

Pierson, K. (1976). Control of walking. Scientific American, 235, 72-86.

RAsch, R. A. (1979). Synchronization in performed ensemble music. Acoustica, 43, 121-131.

RASCH, R. A. (1988). Timing and synchronization in ensemble performance. In J. Sloboda (Ed.), Generative processes in music: The psychology of performance, improvisation, and composition (pp. 70-90). Oxford: Clarendon.

SChIfF, W., Thayer, S. (1968). Cognitive and affective factors in temporal experience: Anticipated or experienced pleasant and unpleasant sensory events. Perceptual \& Motor Skills, 26, 799-808.

TODD, N. P. (1985). A model of expressive timing in tonal music. Music Perception, 3, 33-58.

TodD, R., Boltz, M. G., \& Jones, M. R. (1989). The MIDILAB auditory research system. Psychomusicology, 8, 83-96.

VIDACEK, S., \& WiSHNER, J. (1971). Influence of expectation of task duration on efficiency of muscular activity. Joumal of Applied Psychology, 55, 564-569.

WALSTER, B., ARonson, E. (1967). Effect of expectancy of task duration on the experience of fatigue. Joumal of Experimental Social Psychology, 3, 41-46.

WINTER, D. A. (1983). Biomechanical motor patterns in normal walking. Journal of Motor Balance, 15, 302-330.

Yeston, M. (1976). The stratification of musical rhythm. New Haven, CT: Yale University Press.

\section{APPENDIX \\ The Set of Folk Tunes (Rotsford, 1921) Used in the Pitch Detection Task of Experiment 1}

"A Love Song"' by W. Bynner (China)

"Fond Regrets" by E. Markham (England)

"I'll Have No Other" by J. Mokrejs (Czechia)

"Lithuanian National Hymn"' by W. Griffith (Lithuania)

"Look Out, How It's Raining" by L. Speyer (Austria)

"Near Krakow" by W. A. Roberts (Poland)

"Reapers On the Mountain" by E. Leamy (Ukraine)

"Spite" by A. Mathewson (Italy)

"Why, Oh Mother”' by Edna St. Vincent Millay (Poland)

(Manuscript received October 29, 1992;

revision accepted for publication May 18, 1993.) 